Technological University Dublin

DÜBLIN

ARROW@TU Dublin

2010-01-01

\title{
Photopolymerizable Nanocomposites for Holographic Recording and Sensor Application
}

\author{
Elsa Leite \\ Technological University Dublin \\ Izabela Naydenova \\ Technological University of Dublin, izabela.naydenova@tudublin.ie \\ Svetlana Mintova \\ Laboratoire Catalyse \& Spectrochimie (LCS), ENSICAEN - Université de Caen
}

See next page for additional authors

Follow this and additional works at: https://arrow.tudublin.ie/cieoart

Part of the Condensed Matter Physics Commons, and the Optics Commons

\section{Recommended Citation}

Leite, E. et al. (2010) Photopolymerisable Nanocomposites for Holographic Recording and Sensor Application. Appl.Opt. Vol. 49, No. 19 , 3652-3660. doi:10.1364/A0.49.003652

This Article is brought to you for free and open access by the Centre for Industrial and Engineering Optics at ARROW@TU Dublin. It has been accepted for inclusion in Articles by an authorized administrator of ARROW@TU Dublin. For more information, please contact arrow.admin@tudublin.ie, aisling.coyne@tudublin.ie, gerard.connolly@tudublin.ie.

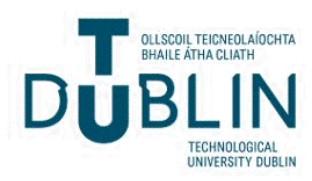




\section{Authors}

Elsa Leite, Izabela Naydenova, Svetlana Mintova, Louis Leclercq, and Vincent Toal 


\title{
Photopolymerizable Nanocomposites for
}

\section{Holographic Recording and Sensor Application}

\author{
E. Leite ${ }^{1}$, I. Naydenova ${ }^{1}$, S. Mintova ${ }^{2}$, L. Leclercq $^{I}$ and V. Toal ${ }^{1}$ \\ ${ }^{1}$ Dublin Institute of Technology, Kevin Street, Dublin 2, Ireland \\ ${ }^{2}$ Laboratoire Catalyse \& Spectrochimie (LCS), ENSICAEN - Université de Caen - CNRS \\ 6, Boulevard du Maréchal Juin 14050 Caen Cedex, France \\ *corresponding authors: elsa.leite@student.dit.ie ; izabela.naydenova@dit.ie
}

Novel nanocomposites consisting of a water soluble acrylamide-based photopolymer and colloidal zeolite nanoparticles of zeolite Beta and zeolite A were prepared. The interactions between the photopolymer components and zeolite nanoparticles in the photopolymerizable nanocomposites were characterized for the first time by ${ }^{13} \mathrm{C}$ NMR and Visible spectroscopy. It was found that the zeolite Beta nanoparticles (up to 5\% wt.) behave as a non-inert additive, resulting in an effective increase in layer thickness that causes doubling of the diffraction efficiency of the nanocomposite in comparison to that of the undoped photopolymer. On the other hand, the nanocomposite containing zeolite A nanoparticles showed no evidence of interaction with the polymer matrix and had similar values of diffraction efficiency and for small addition of nanoparticles (up to $2.5 \%$ wt.) showed slightly higher light induced refractive index modulation of the grating when compared to the undoped photopolymer. The good optical compatibility between the zeolites nanoparticles and the polymer allows a versatile design of photopolymerizable nanocomposites with different properties by selecting the adequate type of zeolite. The nanocomposite containing zeolite Beta nanoparticles demonstrates selective sensing behavior towards toluene and can be coated in either glass or plastic substrates and exposed directly to the environmental conditions.

OCIS Codes: 090.7330, 160.5335 


\section{Introduction}

Photopolymers are used as photosensitive holographic recording materials for holographic optical memories [1] and holographic sensing [2]. Compared to other types of holographic recording materials, photopolymers present many advantages such as self-development, wide spectral sensitivity and relatively low cost [3].

In most cases, the photopolymers used as recording materials contain one or more monomers and a photoinitiator typically consisting of two components, a sensitizing agent and an electron donor. The photopolymer components can be spatially redistributed when illuminated by an optical interference pattern created by two or more mutually coherent laser beams, resulting in a holographic diffraction grating. The layer regions exposed to bright fringes will be composed mainly of polymer (in the case of polyacrylamide with a refractive index, $n=1.5$ ) while those exposed to the dark fringes will be depleted of monomer through concentration gradient driven diffusion and thus have lower refractive index.

Different classes of volume holographic recording materials such as organically modified silica glass [4], sol-gel materials containing zirconium isopropoxide [5] and photopolymerizable nanocomposites containing solid nanoparticles such as $\mathrm{SiO} 2$ [6], $\mathrm{TiO} 2$ [7] , $\mathrm{ZrO} 2$ [8, 9] as dopants, have been developed in the past decade and show improved holographic properties such as higher dynamic range and lower level of shrinkage during holographic recording and hence they have been studied mainly for applications such as holographic memories. These materials are usually sandwiched between two glass slides, which limit their area of exposure to the surrounding environment conditions, which can be a disadvantage if the intended use is for sensing chemical compounds present in the atmosphere. 
The dark diffusion mechanisms in photopolymerizable materials have been studied both experimentally [10] and theoretically by several authors [11, 12]. In the particular case of photopolymerizable nanocomposites, Tomita et al. [13] have proposed that the photo-insensitive nanoparticles, which they assume to be an inactive component, undergo diffusion from the bright to the dark regions. Meanwhile, as monomer is polymerized in bright regions, unused monomer diffuses from dark to bright regions driven by the resulting concentration gradient. These polymerization-driven mutual diffusion processes essentially continue until the photopolymerisation is completed. It was assumed that the inorganic nanoparticles are inert and take part only in the mass transport mechanism during holographic exposure. Recently, Goldenberg et al. [14] have observed that inorganic gold nanoparticles are not inert and promote monomer spatial segregation in addition to mass transport effects observed in the nanocomposite.

In our group, two types of nanocomposites containing colloidal zeolite Beta (BEA-type structure) and zeolite A (LTA-type structure) [15] were prepared. Both types of microporous nanoparticles are zeolites, a class of crystalline aluminosilicalites having a three- dimensional porous structure arising from corner-sharing $\mathrm{SiO}_{4}$ and $\mathrm{AlO}_{4}$ tetrahedra [16]. It was spatially redistributed as a result of holographic recording [17]. The redistribution effect in combination with the nanoparticles ability to adsorb targeted chemical substances makes this nanocomposite a suitable candidate for the design of holographic sensors [18]. Moreover, thin films based on zeolites A and Beta have proved to be effective materials for sensing purposes [19, 20]. High sensitivity, good reversibility and long life of zeolite A-based sensors were demonstrated for detection of water at low concentrations. The Beta films showed a higher sorption capacity towards water vapor than the zeolite A films [20].

Zeolite Beta is also a promising material for the adsorption of toluene and propene [21, 22] Using toluene as a probe test our aim is to detect volatile organic compounds by the change of the diffraction efficiency of holographic sensors. 
This paper reports on development of nanocomposite holographic recording materials in the form of an acrylamide-based photopolymer containing zeolite nanoparticles exhibiting properties suitable for sensing applications. The interactions between the host photopolymer components and zeolite nanoparticles are studied by UV-Visible spectroscopy and ${ }^{13} \mathrm{C}$ NMR spectroscopy. The advantage in the development of this class of volume holographic materials is the fact that the permeable polymer can be coated either in a glass or plastic substrates and exposed directly to the environment atmospherically conditions.

\section{Experimental section}

\subsection{Materials}

The photosensitive nanocomposite material consists of a solution of acrylamide-based photopolymer and zeolite A and Beta nanoparticles. Zeolite Beta is a large-pore microporous material characterized by three sets of mutually perpendicular channels with 12-membered ring apertures [23] (pore sizes of $6.4 \times 7.6 \AA$ and $5.5 \times 5.5 \AA$ ), while zeolite A is a small-pore material with a pore diameter size of $4.2 \AA[15]$.

The photopolymer composition for holographic recording was described previously [24]. In this study $2.0 \mathrm{ml}$ of triethanolamine (TEA) (Sigma) was added to $9 \mathrm{ml}$ of a polyvinyl alcohol (PVA) solution (20\% wt.) (Sigma), then the monomers were added, i.e. $0.6 \mathrm{~g}$ of acrylamide (AA) (Aldrich) and $0.2 \mathrm{~g}$ of $\mathrm{N}, \mathrm{N}$ '-methylenebisacrylamide (BA) (Sigma). The final solution was stirred for 20 minutes, and $4 \mathrm{ml}$ of erythrosine B solution ( $0.11 \%$ wt.) (Aldrich) were added and the resulting mixture was stirred for additional 10 minutes. 
The nanosized zeolite crystals were synthesized from clear precursor suspensions containing organic templates, tetramethylammonium hydroxide $\left(\mathrm{TMA} \cdot 5 \mathrm{H}_{2} \mathrm{O}\right)$ in the case of zeolite LTA and tetraethylammonium hydroxide $(\mathrm{TEAOH})$ in the case of zeolite BEA. The synthesis procedures for zeolite A and Beta are described in detail in Refs. 25 and 26, respectively. The crystalline nanoparticles were extracted from the reaction mixture by a three-step centrifugation $(20,000 \mathrm{rpm}$ for $180 \mathrm{~min}$ ) and subsequently redispersed in distilled water, to obtain stabilized zeolite suspensions in water with a solid concentration of $1 \%$ wt. Dynamic light scattering (DLS) measurements using a Malvern Zetasizer Nano ZS were carried out to determine the mean hydrodynamic diameter of the crystalline zeolite particles. The morphology of the crystals and film thickness were characterized using scanning electronic microscopy (SEM, Philips XL 40).

Before use, the aqueous nanoparticle suspensions were sonicated for 20 minutes in order to obtain a homogeneous particle size distribution and to redisperse the agglomerates. These suspensions were added to the photopolymer solution and the mixture was sonicated for further 10 minutes. Different concentrations of nanoparticles were introduced in the dry layers (Table 1). In order to obtain similar viscosities of the solutions containing different concentrations of nanoparticles, deionised water was added to the photopolymer solutions. Dynamic Light Scattering measurements of the suspensions were performed immediately and after again for 24 hours to check their stability.

The photopolymerizable nanocomposite films were prepared by spreading $0.4 \mathrm{ml}$ of the suspensions on glass plates with dimensions of $26 \times 38 \mathrm{~mm}^{2}$ followed by drying on an optical table for 24 hours.

Toluene was purchased from Sigma. 


\subsection{Measurements}

Unslanted volume holographic transmission gratings were recorded by exposure of the holographic recording material to two mutually coherent s-polarized beams of wavelength $532 \mathrm{~nm}$. Total exposure energy was $600 \mathrm{mJcm}^{-2}$ at a spatial frequency of $1000 \mathrm{lmm}^{-1}$. The grating growth was monitored in real time by probing with an unexpanded He-Ne laser of wavelength $633 \mathrm{~nm}$ incident at the Bragg angle. The sample was held on a rotation stage, allowing the grating angular selectivity to be measured, at the completion of exposure, by adjusting the incident angle of the probe beam. The angular selectivity curve was used to calculate the effective thickness of the grating. This parameter and the maximum diffraction efficiency, $\eta$, (here defined by the ratio of the intensity of the first diffraction order and the incident intensity of the probe beam) were used to calculate the refractive index modulation amplitude, $n_{l}$, using Kogelnik's coupled wave theory [27]:

$$
n_{1}=\frac{\lambda \cos \theta \arcsin \sqrt{\eta}}{\pi d}
$$

where $\lambda$ is the reconstructing beam wavelength, $\theta$ is the reading beam incidence angle and $d$ is the thickness of the photosensitive layer.

The absorption spectra of solutions were recorded using a Perkin-Elmer Lambda 900 UV/VIS/NIR spectrometer. Solutions of erythrosine B in water, photopolymer and water solutions containing only one of the matrix components (PVA 10\%, acrylamide or TEA) were spectrally characterized.

The solid-state ${ }^{13} \mathrm{C}$ MAS H-decoupled NMR measurements of the samples were performed using a Bruker Biospin 400 spectrometer operating at a resonance frequency of $100.62 \mathrm{MHz}$. All spectra were recorded at a spinning rate of $12 \mathrm{kHz}$, recycle delay of $60 \mathrm{~s}$ and pulse length of $3.8 \mu \mathrm{s}$. 
In order to evaluate the sensing properties of the materials, recorded volume gratings of acrylamide based photopolymer and Beta nanocomposite were exposed to toluene vapors (and their efficiency was measured again by means of the angular selectivity curve. The gratings were placed in a container into which toluene was added and the container sealed for the duration of the exposure (10 minutes). This experiment was carried out in a fume hood, and contact from ignition sources was prevented. The concentration of toluene inside the volume of the container was estimated to be $19 \mathrm{ppm}$.

\section{Results and Discussion}

\subsection{Characterization of photopolymerizable nanocomposites}

The morphology of the zeolite nanocrystals (A and Beta) prior to mixing with the photopolymer solutions was characterized by DLS and SEM. (Figs. 1 and 2) As can be seen, the size of the crystals is below $100 \mathrm{~nm}$ for both types of zeolite, and both materials exhibit monomodal particle distribution. Zeolite Beta and A have spherical and almost cubic shape, respectively (Figure $1)$.

The size of the zeolite particles and their stability in the photopolymer suspensions were characterized by measuring the DLS curves for the photopolymerizable nanocomposites. No aggregation of the zeolite nanoparticles directly after mixing and after $24 \mathrm{~h}$ aging was observed (Figure 2). These results demonstrate that the zeolite nanoparticles are compatible with the photopolymer, thus homogeneous coating suspensions and dry layers are obtained.

Further, the surface morphology of the films was studied using SEM. The SEM picture taken from the film with zeolite Beta loading of $5 \mathrm{wt} . \%$ is shown in Figure 3a. It can be seen that the nanoparticles are randomly and homogeneously distributed within and at the surface of the film. The thickness of the film is about $50 \mu \mathrm{m}$ as estimated from the scratch (white portion shows the glass 
substrate) in the SEM picture (Figure 3a). Moreover, the zeolite photopolymerizable nanocomposite containing both types of zeolite particles yield good optically transparent films which are thus suitable for optical applications. This is further supported by Figure 3b, which shows an acrylamide based photopolymer containing zeolite A nanoparticles (5\%wt.) assembled in film on glass support.

\subsection{Holographic properties of photopolymerizable nanocomposites}

The holographic properties, i.e., diffraction efficiency, thickness and refractive index modulation of the photopolymerizable nanocomposites assembled in the films with different concentrations of zeolites Beta and A were studied. As can be seen from Figure 4b, the diffraction efficiency $(\eta)$ of the gratings for Beta-loaded nanocomposites increases with the concentration of the particles up to $5 \mathrm{wt} . \%$. The maximum value of $\eta$ is about $35 \%$ for the film with a thickness of $40 \mu \mathrm{m}$ Figure $4 \mathrm{a}$ (squares). The thickness of the films increases from 20 to $50 \mu \mathrm{m}$, as the concentration of zeolite Beta increases from 0 to 5 wt.\%. However the thickness of the films doped with zeolite A nanocrystals is constant at $30 \mu \mathrm{m}$. There is a slight improvement of the refractive index modulation for the films with small additions of zeolite A nanoparticles (Figure $4 \mathrm{c}$ - circles) and overall the refractive index is higher than in the case of addition of the same concentration of Beta nanoparticles (Figure $4 \mathrm{c}$ - circles).

\subsection{Influence of the matrix on grating formation in photopolymerizable nanocomposites}

To determine how the presence of zeolite nanoparticles affects the holographic properties of acrylamide based photopolymer, we compared the recording characteristics of layers having different concentrations of polymer components. Layers were prepared using stock solutions with 5\%, 10\% and 20\% wt. polyvinyl alcohol (PVA). The compositions of the different suspensions are

presented in Table 2. The refractive index modulation determined for all samples are shown in Figure 5. 
The layers showed lower refractive index modulation with increasing PVA concentration, which is expected since monomer concentration in the dry layer is $20 \pm 0.8 \%$ wt. in the case of 5 $\%$ PVA stock solution, $16 \pm 0.6 \%$ wt. for $10 \%$ PVA stock solution and $12 \pm 0.3 \%$ wt. for $20 \%$ PVA stock solution. These standard deviations account for similar monomer concentrations when using the concentration of the PVA stock solution. Despite of that fact, we have observed different rates of decrease of the refractive index modulation with the increasing concentration of nanoparticles for the two nanocomposites. For the nanocomposite containing Beta (figure 5a) the decrease is greater than the one observed in layers containing the same percentage of zeolite A (figure 5b).

Since the observed difference in the decrease in the refractive index modulation for the doped and undoped layers can not be accounted by differences in the monomer concentration, one can suspect that it could be caused by the TEA presence. There is around a $10 \%$ TEA concentration difference in the nanocomposites concentration prepared with $20 \%$ PVA stock solution and with 5\% PVA stock solution. This hypothesis was investigated in the next section.

\subsection{Interactions between photopolymer components and zeolite nanoparticles studied by visible spectroscopy}

The interactions between zeolite nanoparticles (A and Beta) and the photopolymer components were studied by means of Visible Spectroscopy. Typical absorption spectra are shown in Figure 6a for aqueous solutions of pure erythrosine B (sensing dye) and a mixture of erythrosine B and zeolites. No shift in the absorption spectra of the dye was observed after the addition of the zeolite nanoparticles indicating the absence of interaction between the dye and the two types of nanoparticles. 
Spectra were also taken from photopolymer solutions. The shapes of the spectra remained the same but a red shift of maximum of absorption peak with $9 \mathrm{~nm}$ and $10 \mathrm{~nm}$ in the case of solutions with Beta and A, respectively is measured. This is expected since the absorption maxima of dyes are dependent on solvent polarity. In non-hydrogen-bond donating solvents, solvation of dye molecules probably occurs via dipole-dipole interactions, whereas in hydrogen-bond donating solvents the phenomenon is more hydrogen bonding in nature [28, 29]. Studies in xanthenes dyes such as eosin and erythrosin showed that the maximum absorption peak in aqueous solutions shifts to higher wavelengths in ethanol, and the same type of solvent dependency of the absorption spectra was observed for hidroxyxanthenes, in which the shift in the absorption maximum is due to hydrogen bonding [30].

Different components of the photopolymer (one at a time) were added to the solution of zeolite and erythrosine B (Figure 6b). It was observed that the addition of acrylamide to the water dispersion of zeolite nanoparticles and erythrosine B dye leads to a $2 \mathrm{~nm}$ red shift in the erythrosine B absorption peak in both solutions containing zeolite Beta and A nanoparticles. This indicates that a similar change of the solvent polarity after acrylamide additions occurs in both solutions. A red shift of $5 \mathrm{~nm}$ in the absorption peak for zeolite Beta was detected in the presence of TEA but there was no change in the case of zeolite A solution. This indicates that there is a difference in the interaction between the two types of nanoparticles and the TEA molecules. Both solutions (water dispersions of Beta and zeolite A) showed a larger red shift in the absorption peak of erythrosine B in presence of PVA (16 $\mathrm{nm}$ in the case of zeolite Beta and $12 \mathrm{~nm}$ for zeolite A).

\subsection{Interactions between photopolymer components and zeolite nanoparticles studied by NMR}

To further clarify the nature of the difference between the two nanocomposites layers of acrylamide based photopolymer with $5 \%$ of Beta and zeolite A nanoparticles were studied by ${ }^{13} \mathrm{C}$ NMR (Carbon Nuclear Magnetic Resonance) and compared with undoped layers (Figure 7). In this 
spectroscopic technique the interaction of the nuclei of carbon isotopes with a static magnetic field can be studied and one should expect to distinguish organic molecules incorporated or adsorbed in the zeolite framework on the basis of their chemical shifts in the ${ }^{13} \mathrm{C}$ NMR spectra.

In Beta containing nanocomposite the two peaks corresponding to TEA are shifted to lower field as highlighted by the arrows in Figure 7a indicating an interaction (possibly hydrogen bonding)

between the TEA and the nanoparticles. However, no shift in the ${ }^{13} \mathrm{C}$ NMR peaks is observed in the zeolite A nanocomposite. The spectra were collected outside and inside grating area. As can be seen a more pronounced shift in the $\mathrm{C}$ peak is obtained for Beta-photopolymer inside the grating, thus confirming the strong interactions between the particles and the photopolymer.

\subsection{Photopolymerizable nanocomposites for sensing of toluene}

Unslanted volume holographic transmission diffraction gratings in photopolymerizable materials recorded as described in section 2.2 were tested as sensors [11]. The principle of operation of this type of sensor is based in a change of the diffraction efficiency $(\eta)$.

Diffraction efficiency can be obtained from Eq. 1:

$$
\eta=\sin ^{2}\left(\frac{\mathbf{n}_{1} \pi \mathbf{d}}{\lambda \cos \theta}\right)
$$

Deriving equation 2 we can estimate the change in diffraction efficiency:

$$
\Delta \eta=\sin \left(\frac{2 n_{1} \pi d}{\lambda}\right) \frac{\pi}{\cos \theta \lambda}\left[d \Delta n_{1}+n_{1} \Delta d-\left(\frac{n_{1} d}{\lambda}\right) \Delta \lambda\right]
$$


where $n_{1}$ is the refractive index modulation, $d$ is the thickness of the layer, $\lambda$ is the wavelength of light, $\theta$ is the angle between the two recording beams.

A change in the diffraction efficiency is caused by a change of a thickness $(\Delta d)$, which is due to shrinkage/ swelling of the layer or is caused by the redistribution of the nanoparticles during the recording of the holographic diffraction gratings.

Chemical vapors such as acetone $(n=1.36)$, chloroform $(n=1.45)$ and toluene $(n=1.50)$ have been reported [31] to change the overall average refractive index of a reflection grating recorded in undoped acrylate polymer. Most importantly, this is achieved in the case of transmission gratings, where the diffraction efficiency does not depend on this parameter.

Both undoped acrylamide and Beta photopolymerizable nanocomposite were exposed to toluene and their angular selectivity curves were obtained before and after exposure (Figure 8) and the results are summarized in Figure 9. As can be seen, the nanocomposite grating exhibits a greater change in diffraction efficiency than the undoped photopolymer grating. For gratings made in undoped photopolymer with initial diffraction efficiency ranging from 20 to $80 \%$ a change in the diffraction efficiency of less than $10 \%$ occurred after exposure to toluene (maximum observed was $\Delta \eta=9.8 \%$ for a grating with initial diffraction efficiency of $58 \%$ ). As discussed previously, the change in the diffraction efficiency in undoped layers is possibly due to a toluene induced dimensional change of the layer since one should not expect a change in the refractive index modulation. More detailed studies are in progress in order to identify the exact mechanism of diffraction efficiency change of transmission gratings in undoped layers exposed to toluene. The gratings recorded in the composite layers containing $5 \%$ Beta nanoparticles, exhibit a maximum diffraction efficiency change of $22 \%$ (double of the value observed for undoped layers) in a range of initial diffraction efficiencies ranging from 20 to $60 \%$. An improvement in the sensitivity towards toluene for Beta- photopolymer composite (Figure 9) is observed. It is also worth noting that the 
higher the initial diffraction efficiency of the doped layers is, the greater change caused by the exposure to toluene. This can be explained by the fact that in higher diffraction efficiency gratings the redistribution of nanoparticles is more effective, - that is, there is a greater difference between the concentrations of nanoparticles in the bright and dark regions. It is also expected that the refractive index in the bright regions is higher than the refractive index in the dark regions rich in zeolite nanoparticles. Due to adsorption of toluene in the nanocomposite grating, the refractive index of the dark regions is increased. Thus the resulting refractive index modulation which is defined as the difference between the refractive index in the bright and in the dark regions decreases. This ultimately is observed as a decrease of the measured diffraction efficiency. The observed decrease is expected to be higher when the nanoparticles redistribution is more effective, which is confirmed by the experimental data shown in Figure 9.

\section{Conclusions}

Zeolite-photopolymer nanocomposites with good optical quality were prepared by combining different concentrations of zeolite nanoparticles (A and Beta) with various concentrations of a photopolymer binder. The addition of zeolite Beta to an acrylamide based photopolymer leads to an interaction between the nanoparticles and the TEA photopolymer components. However, this interaction does not occur in the case of zeolite A nanoparticles, which can be explained by the high hydrophilicity and small pores of this zeolite. The Beta nanoparticles act as non-inert components during holographic recording, bonding to the triethanolamine (TEA) electron donor molecules, while no evidence of these interactions was found regarding zeolite A. These interactions could be responsible for the different holographic characteristics observed for the two types of nanocomposites. Firstly the layer thickness increases and secondly the diffraction efficiency is almost doubled (5\% wt. BEA concentration) in comparison with the undoped photopolymer. This effect is exploited for the fabrication of holographic sensors. The addition of 5 wt. \% Beta to the 
polymer layers yields to an increase of the transmission grating sensitivity towards toluene in comparison to the undoped photopolymer.

This work was supported by the Technological Sector Research: Strand I - Post-Graduate R\&D Skills Programme. The authors acknowledge the Facility for Optical Characterization and Spectroscopy at Dublin Institute of Technology for technical support and S. Martin for constructive suggestions.

\section{References}

[1] Gambogi, W.; Weber, A.; Trout, T. "Holographic Transmission Elements Using Improved Photopolymer Films" Proc. SPIE 1993, 2043, 2-13.

[2] Kabilan, S.; Marshall, A.; Sartain, F; Lee, M.-C; Hussain, A.; Yang, X.; Blyth, J.; Karangu, N.; James, K.; Zeng, J.; Smith, D., Domschke, A.; Lowe, C. "Holographic glucose sensors" Biosens. Bioelectron. 2005, 20, 1602-1610.

[3] Naydenova, I.; Sherif, H.; Mintova, S.; Martin, S.; Toal, V. "Holographic recording in nanoparticle-doped photopolymer" Proc. SPIE 2006, 6252, 625206.1-625206.6.

[4] Cheben, P.; Calvo, M. “A photopolymerizable glass with diffraction efficiency near 100\% for holographic storage", Appl. Phys. Lett. 2001, 78, 1490-1492

[5] Del Monte, F.; Martinez, O.; Rodrigo, J.; Calvo, M.; Cheben, P.; “A volume holographic sol-gel material with large enhancement of dynamic range by incorporation of high refractive index species", Adv. Mat. 18, 2006, 18, 2014-2017

[6] Tomita, Y.; Nishibiraki, H.“'Improvement of holographic recording sensitivities in the green in $\mathrm{SiO} 2$ nanoparticle-dispersed methacrylate photopolymers doped with pyrromethene dyes", Appl. Phys. Lett. 2003, 83, 410- 412. 
[7] Sanchez, C.; Escuti, M.;.Heesh, C.; Bastiaansen, C.; Broer, D.; Loos, J; Nussbaumer, R.; “TiO2 Nanoparticle-Photopolymer Composites for Volume Holographic Recording” Adv. Funct. Mat. 2005, 15, 1623-1629.

[8] Sakhno, O.; Goldenberg, L.; Stumpe, J.; Smirnova, T. "Surface modified ZrO2 and TiO2 nanoparticles embedded in organic photopolymers for highly effective and UV-stable volume holograms", Nanotechnology, 2007, 18, 105704

[9] Omura, K.; Tomita, Y.; "Photopolymerization kinetics and volume holographic recording in ZrO2 nanoparticle-polymer composites at 404 nm”, Appl. Phys., 2010, 107, 023107-023112

[10] Martínez-Matos, O.; Calvo, M.; Rodrigo, J.; Cheben, P.; Del Monte, F. "Diffusion study in tailored gratings recorded in photopolymer glass with high refractive index species”, Appl. Phys. Lett. 2007, 91, $141115-141118$

[11] Piazzolla, S.; Jenkins, B. "First-harmonic diffusion model for holographic grating formation in photopolymers," J. Opt. Soc. Am. B 2000, 17, 1147-1157

[12] Babeva, T.; Naydenova, I.; Mackey, D.; Martin, S.; Toal, V. "Two-way diffusion model for shortexposure holographic grating formation in acrylamide-based photopolymer," J. Opt. Soc. Am. B 2010, 27, 197-203

[13] Tomita, Y. Holographic assembly of nanoparticles in photopolymers for photonic applications; SPIE Newsroom: Micro/ Nano Lithography \& Fabrication, 2007.

[14] Goldenberg L.; Sakhno, O.; Smirnova, T.; Helliwell, P.; Chechik V.; Stumpe, J.; "Holographic Composites with Gold Nanoparticles: Nanoparticles Promote Polymer Segregation“, Chem. Materials 2008, 20, 4619-4627.

[15] Baerlocher, C.; McCusker, L.; Olson, D. Atlas of zeolite framework types; Elsevier: Amsterdam, 2007.

[16] Dyer, A.; An introduction to zeolite molecular sieves; John Wiley and sons: Chichester 1988.

[17] Leite, E.; Naydenova, I.; Pandey, N.; Babeva, T.; Majano, G.; Mintova, S.; Toal, V.; "Investigation of the light induced redistribution of zeolite Beta nanoparticles in an acrylamidebased photopolymer", J. Opt. A: Pure Appl. Opt. 2009, 11, 024016. 
[18] Naydenova, I.; Toal, V.; Nanoparticle Doped Photopolymers for Holographic Applications in Ordered Porous Solids: Recent Advances and Prospects by V. Valtchev, S. Mintova and M. Michael Tsapatsis ed. 2008.

[19] Mintova, S.; Mo, S.; Bein, T.; "Humidity Sensing with Ultrathin LTA-Type Molecular Sieve Films Grown on Piezoelectric Devices", Chem. Mater. 2001, 13, 901-905.

[20] Mintova, S.; Bein, T.; "Nanosized zeolite films for vapor-sensing applications", Microp. Mesoporous Mater. 2001, 50, 159-166.

[21] Zhang, Y.; Su, Q.; Wang, Z.; Yang, Y.; Xin, Y.; Han, D.; Yang, X.; Wang, H.; Gao. X.; Zhang, Z. "Synthesis and Toluene Adsorption/Desorption Property of Beta Zeolite Coated on Cordierite Honeycomb by an In Situ Crystallization Method", Chem. Eng. Tech. 2008, 31, 1856-1862.

[22] Burke, N.; Trimma D.; Howe, R.; "The effect of silica:alumina ratio and hydrothermal ageing on the adsorption characteristics of BEA zeolites for cold start emission control”, Appl. Cat. B: Env. 2003, 46, 97-104.

[23] Mintova, S.; Reinelt, M.; Metzger, T.; Senkera, J.; Bein, T.; "Pure silica BETA colloidal zeolite assembled in thin films", Chem. Commun. 2003, 3, 326-327.

[24] Martin, S.; Leclere, P.; Renotte, Y.; Toal V.; Lion, Y.; “Characterisation of an acrylamide-based dry photopolymer holographic recording material", Opt. Eng. 1994, 33, 3942-3946.

[25] Mintova, S.; Olson, N.; Valtchev, V.; Bein, T.; "Mechanism of Zeolite A Nanocrystal Growth from Colloids at Room Temperature", Science 283, 1999, 958-960.

[26] Mihailovab, B.; Valtchev, V.; Mintova, S.; Faust, A.-C.; Petkov, N.; Bein, T.; "Interlayer stacking disorder in zeolite beta family: a Raman spectroscopic study", Phys. Chem. Chem. Phys. 2005, 7, 2756-2763.

[27] Kogelnik, H.; "Coupled-wave theory for thick hologram gratings", Bell. Syst. Tech. J. 1969, 48, 2909.

[28] Reichardt, C.; Solvents and Solvent Effects in Organic Chemistry; Wiley-VCH, 3rd ed.: Germany, 2003. 
[29] Rauf, M.; Soliman A.; Khattab, M; "Solvent effect on the spectral properties of Neutral Red", Chem. Cent J. 2008, 2: 19

[30] Islam, S.; Yoshikawa, Y.; Fujitsuka, M.; Watanabe A.; Ito, O. "Studies on photochemical processes of xanthene dyes by means of the transient absorption spectra in the visible/ near-IR regions” Bull. Chem. Soc. Jpn 1998, 71, 1543-1548

[31] Hsiao, V.; Kirkey, W.; Chen, F.; Cartwright, A.; Prasad, P.; Bunning, T.; "Organic Solvent Vapor Detection Using Holographic Photopolymer Reflection Gratings”, Adv. Mat. 2005, 17, 22112214. 
Table 1 - Compositions used for preparations of photopolymerizable nanocomposite (NC) layers.

\begin{tabular}{lccc}
\hline \multicolumn{1}{c}{ Photopolymerizable } & Photopolymer & Nanoparticle & Water (mL) \\
Material & Solution (mL) & Solution (mL) & \\
\hline Undoped & 1 & 0 & 2.5 \\
Photopolymer & 1 & 0.25 & 2.25 \\
NC BEA 1\% & 1 & 0.625 & 1.875 \\
NC BEA 2.5\% & 1 & 1.25 & 1.25 \\
NC BEA 5\% & 1 & 2.5 & 0 \\
NC BEA 10\% & 1 & 0.2 & 2.3 \\
NC LTA 1\% & 1 & 0.5 & 2.0 \\
NC LTA 2.5\% & 1 & 1.0 & 1.5 \\
NC LTA 5\% & 1 & 2.0 & 0.5 \\
NC LTA 10\% & & & \\
\hline
\end{tabular}


Table 2 - Composition of photopolymerizable layers (dry content wt. \%) prepared with different concentrations of matrix components: undoped layer (1); nanocomposite containing concentration 1 of zeolite (2) and nanocomposite containing concentration 2 of zeolite (3); Zeolite is either Beta or A.

\begin{tabular}{|c|c|c|c|c|c|c|c|c|c|}
\hline \multirow{2}{*}{$\begin{array}{c}\text { Matrix } \\
\text { component }\end{array}$} & \multicolumn{3}{|c|}{ Matrix with 20\% PVA } & \multicolumn{2}{c|}{ Matrix with 10\% PVA } & \multicolumn{3}{c|}{ Matrix with 5\% PVA } \\
\cline { 2 - 10 } & 1 & 2 & 3 & 1 & 2 & 3 & 1 & 2 & 3 \\
\hline PVA & $54 \%$ & $54 \%$ & $51 \%$ & $37 \%$ & $36 \%$ & $34 \%$ & $57 \%$ & $56 \%$ & $52 \%$ \\
\hline TEA & $34 \%$ & $33 \%$ & $32 \%$ & $46 \%$ & $46 \%$ & $43 \%$ & $23 \%$ & $22 \%$ & $20.5 \%$ \\
\hline AA & $9 \%$ & $9 \%$ & $9 \%$ & $13 \%$ & $12 \%$ & $11.5 \%$ & $15 \%$ & $15 \%$ & $14 \%$ \\
\hline BA & $3 \%$ & $3 \%$ & $3 \%$ & $4 \%$ & $4 \%$ & $4 \%$ & $5 \%$ & $5 \%$ & $4.5 \%$ \\
\hline Zeolite & - & $1 \%$ & $5 \%$ & - & $2 \%$ & $7.5 \%$ & - & $2 \%$ & $9 \%$ \\
\hline
\end{tabular}



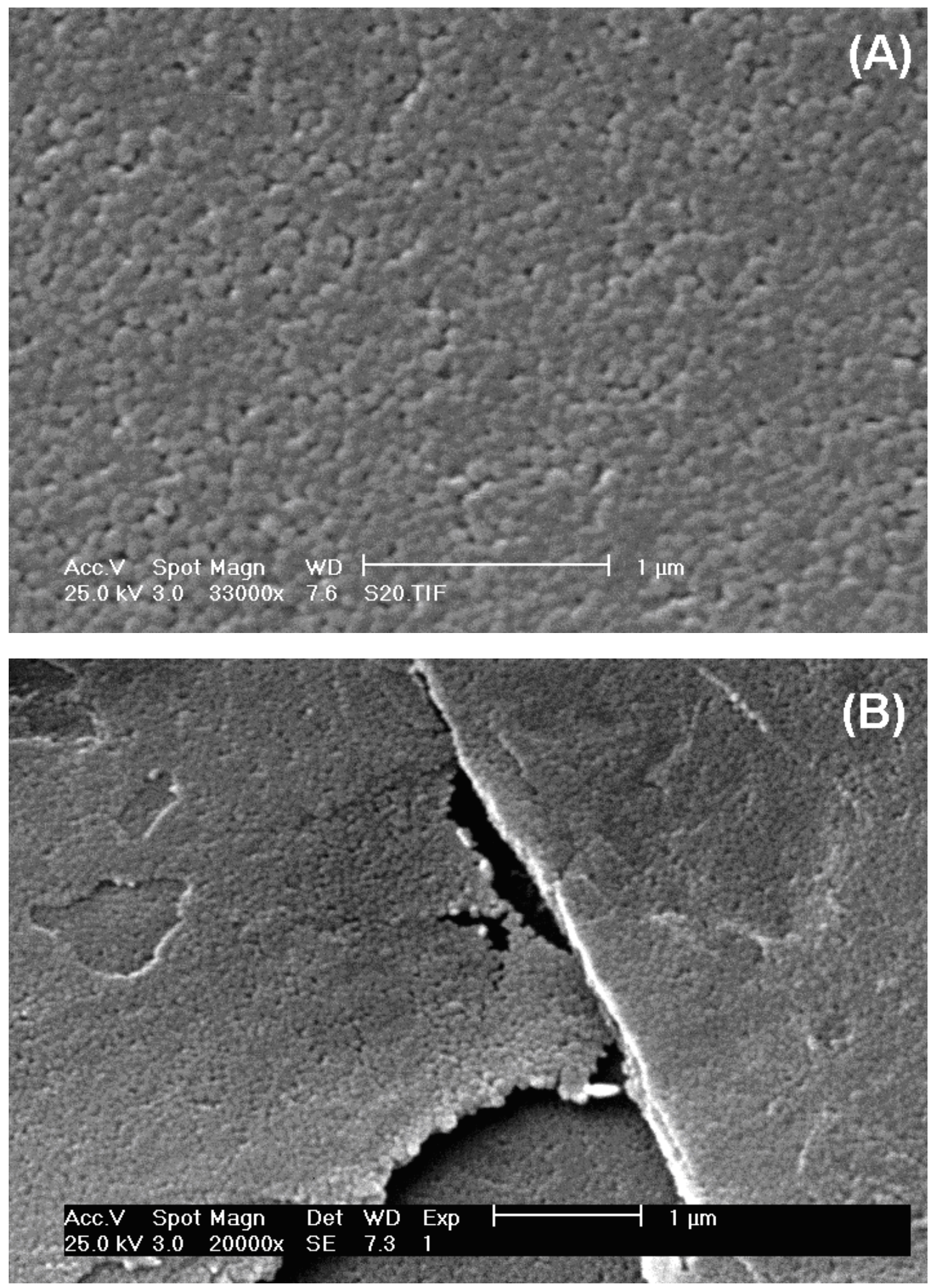

Figure 1. SEM pictures of (A) Zeolite Beta and (B) Zeolite A nanoparticles 


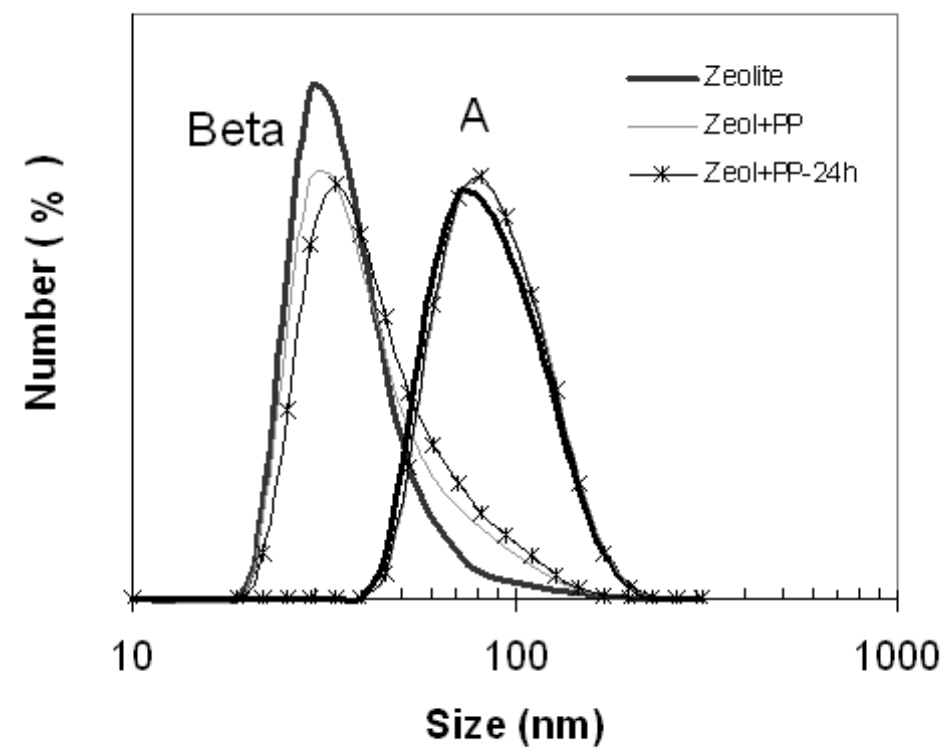

Figure 2. Dynamic Light Scattering curves (number weighted) of pure zeolite suspensions (black), acrylamide based photopolymer and zeolite nanoparticles freshly mixed (grey) and after 24 hours at ambient conditions (grey with stars) 

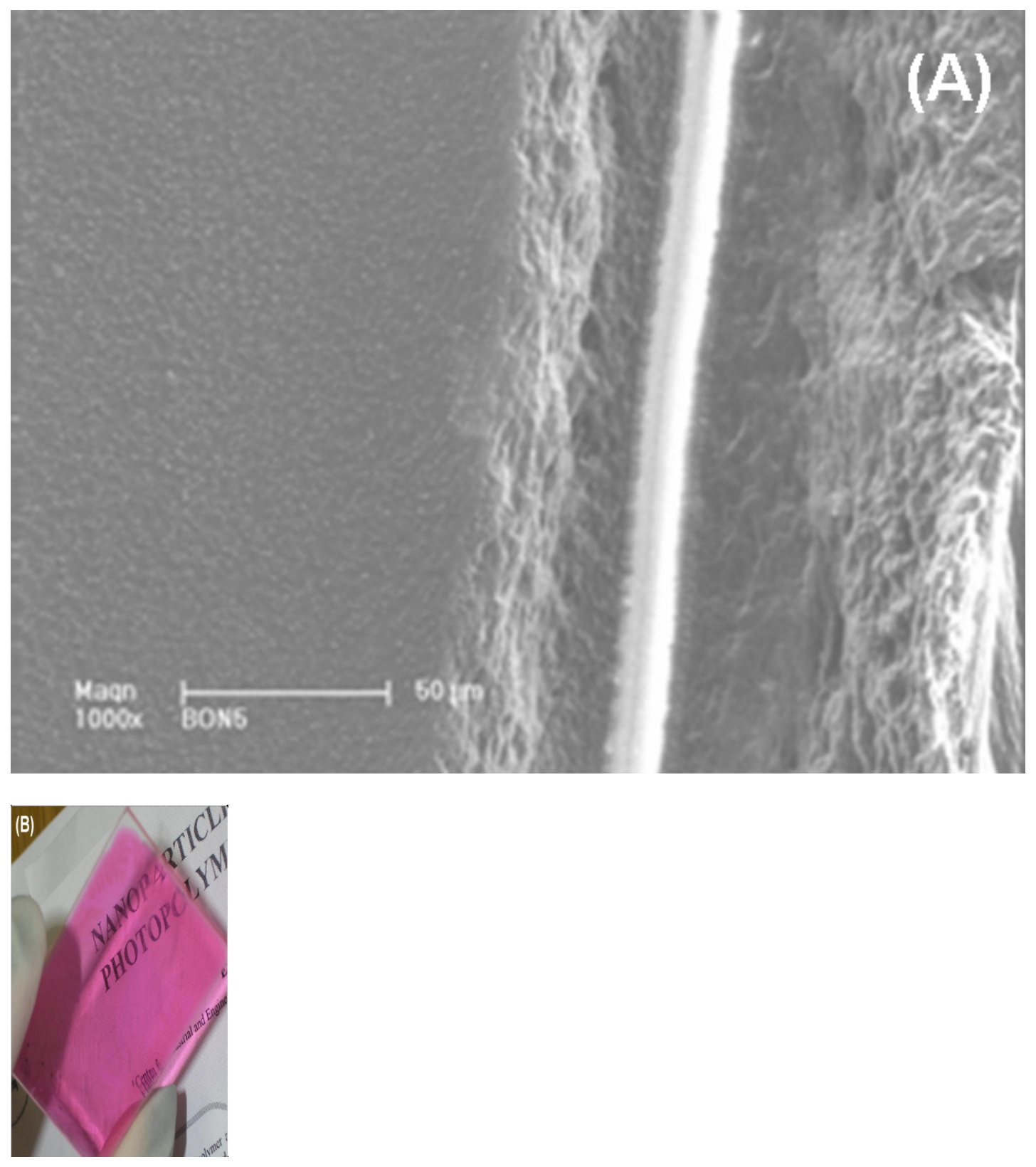

Figure 3. (A) SEM of the acrylamide based photopolymer layer (50 $\mu \mathrm{m}$ thickness) doped with Beta nanoparticles (5 wt. \%); the scale bar is $50 \mu \mathrm{m}$ (B) Optically transparent film of acrylamide based photopolymer doped with zeolite A nanoparticles (5 wt. \%) 

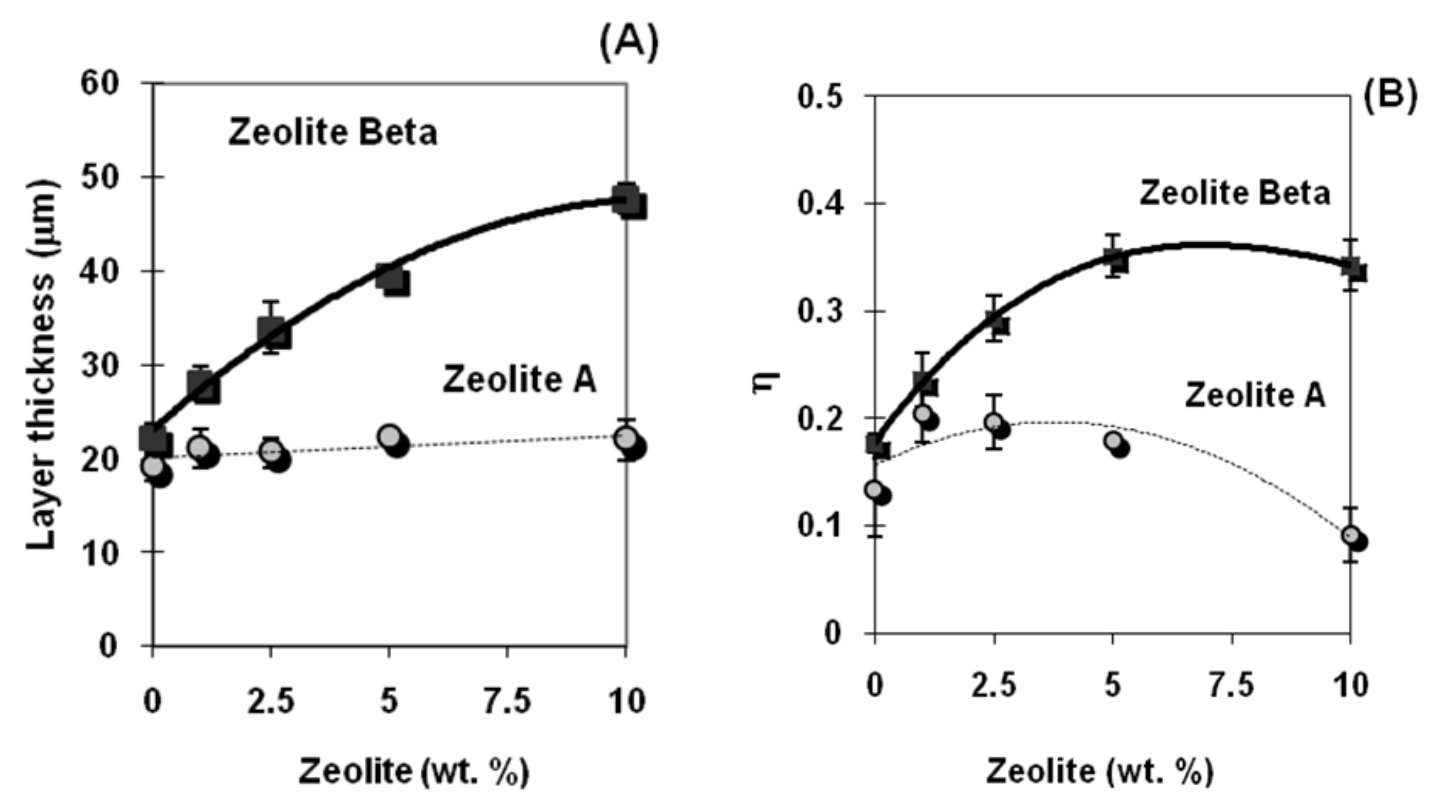

(C)

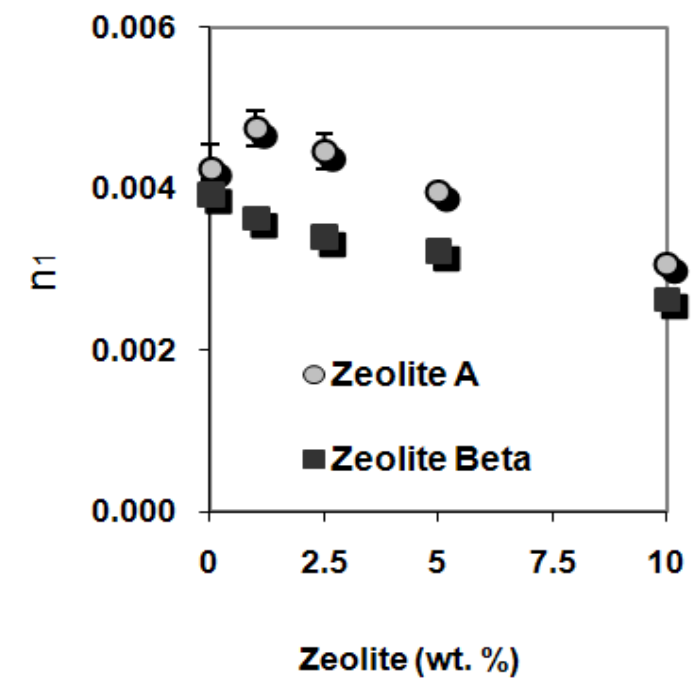

Figure 4. Holographic properties of zeolite nanocomposites: (A) thickness of layers, (B) diffraction efficiency, and (C) refractive index modulation; Gratings recorded at $1000 \mathrm{lmm}^{-1}$, exposure energy of $600 \mathrm{mJcm}^{-2}$ and using $20 \%$ PVA solution. 


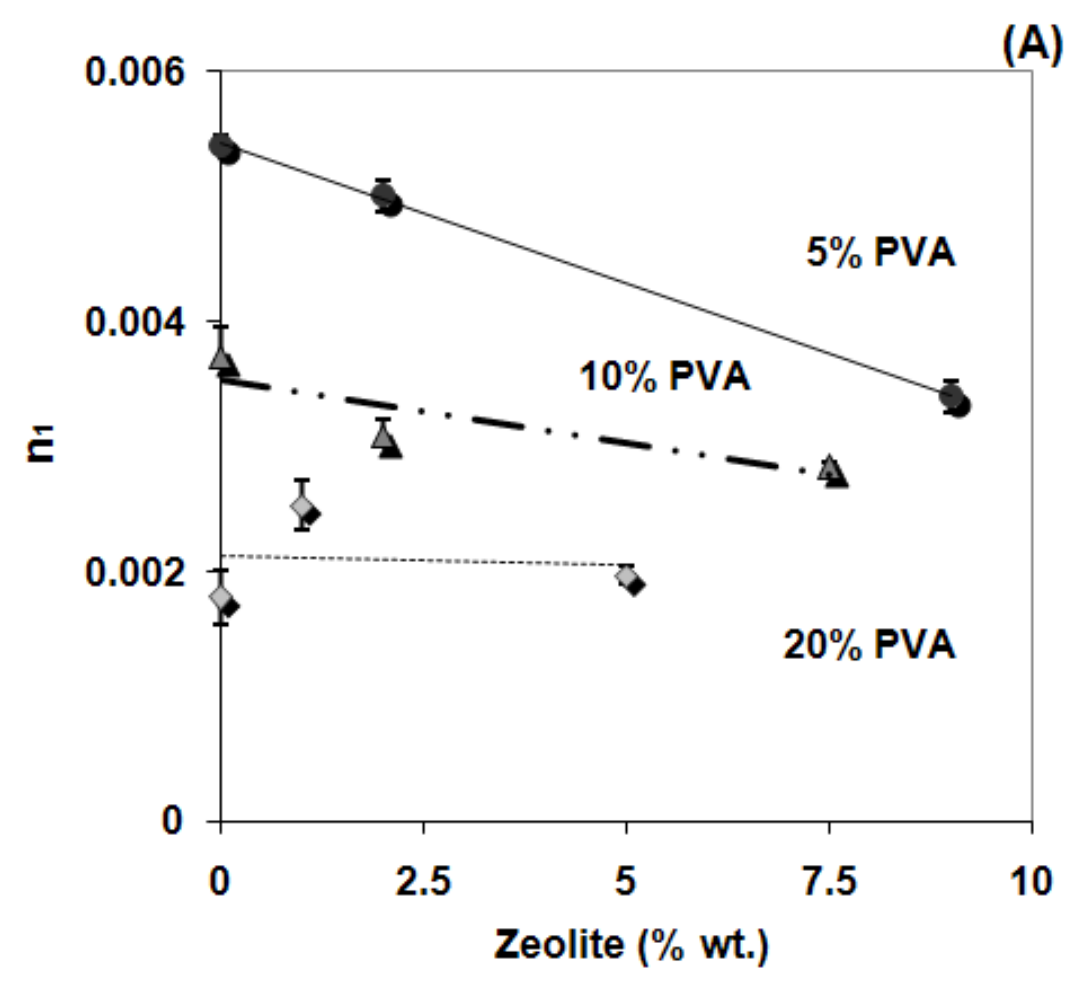

(B)

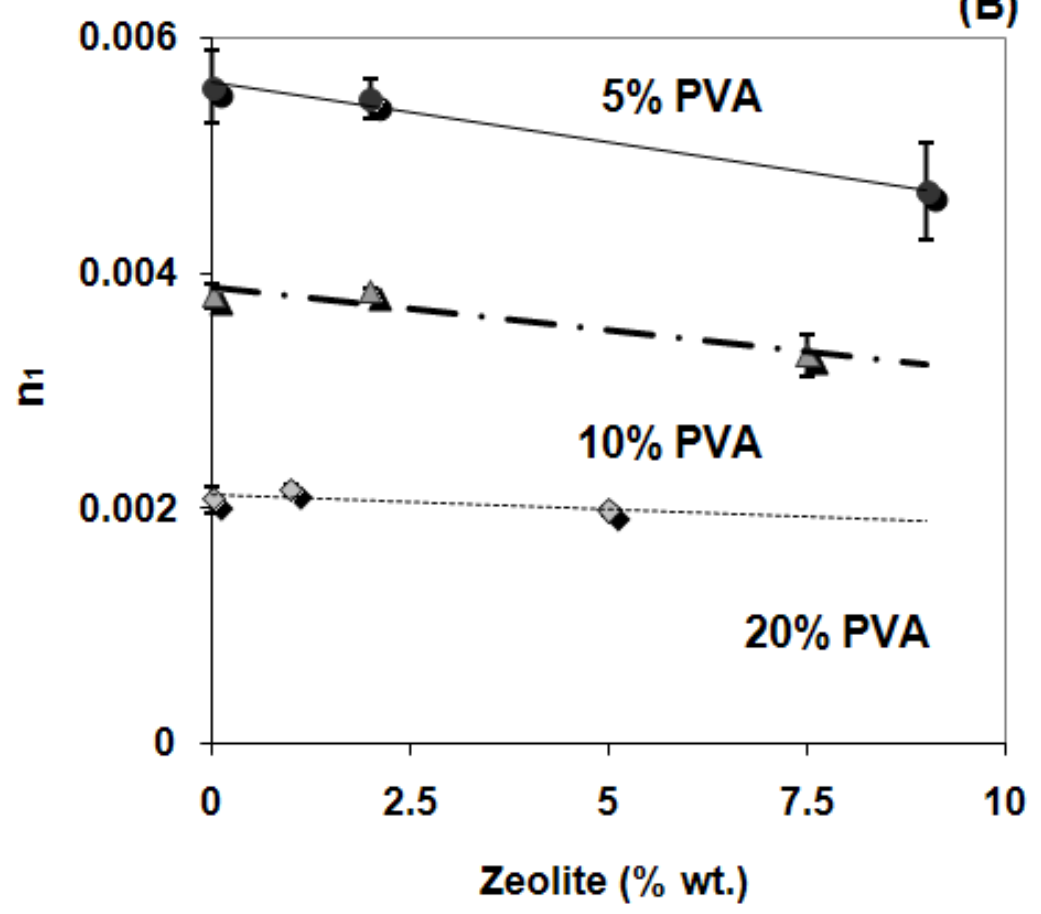

Figure 5. Refractive index modulation of acrylamide based photopolymer nanocomposites containing nanoparticles of (A) zeolite Beta (B) zeolite A. The layers were prepared with PVA stock solutions with a concentration of $5 \%$ PVA, $10 \%$ and $20 \%$ wt. 

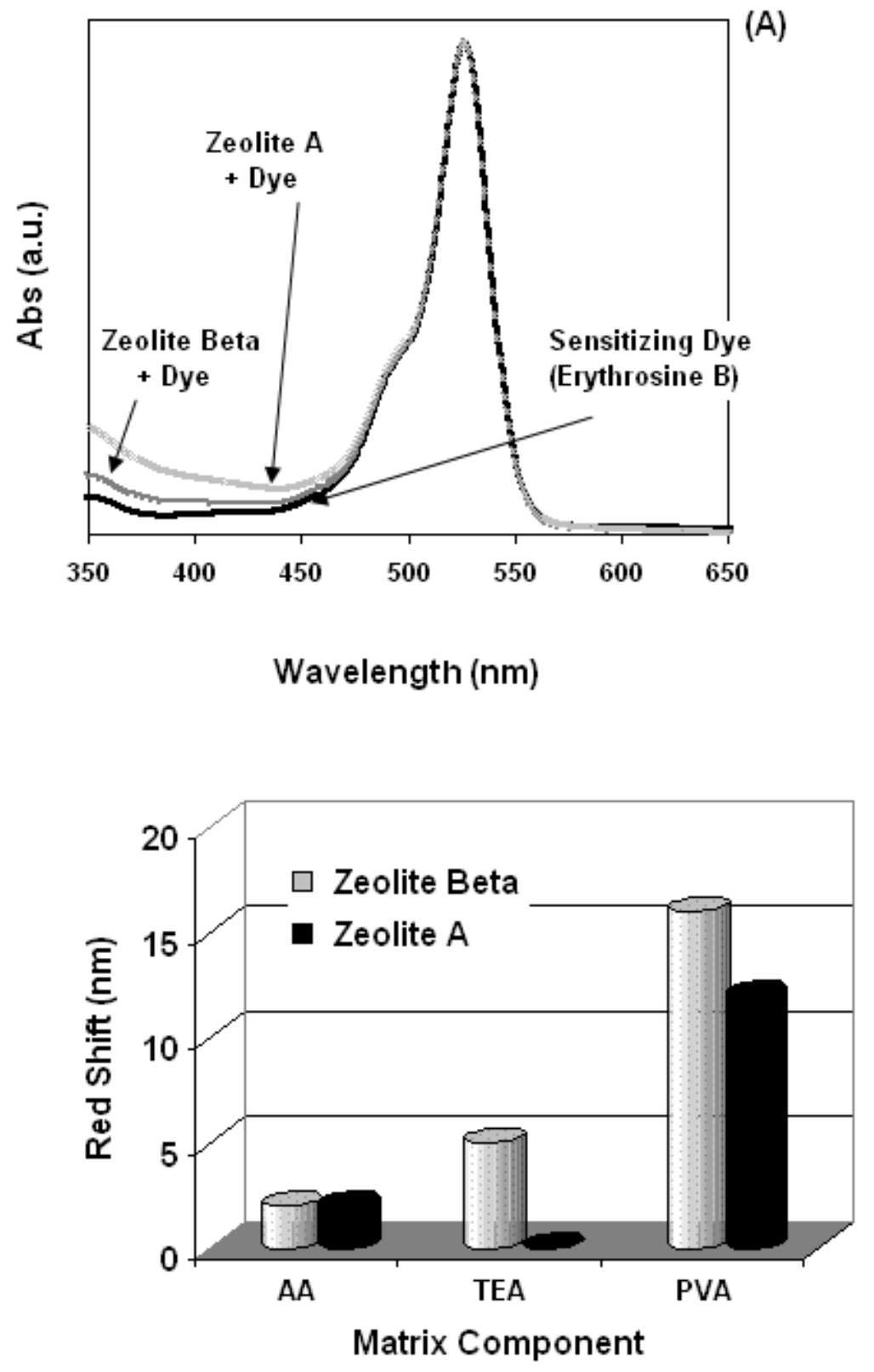

(B)

Figure 6. (A) Visible absorption spectra of erythrosine B in water (black), aqueous solution of zeolite A (light grey) and aqueous solution of zeolite Beta (dark grey), (B) Change in the position of the absorption maximum in aqueous solutions containing erythrosine B and zeolite Beta or A when compared to aqueous solution in the presence of acrylamide - AA, TEA and PVA. 


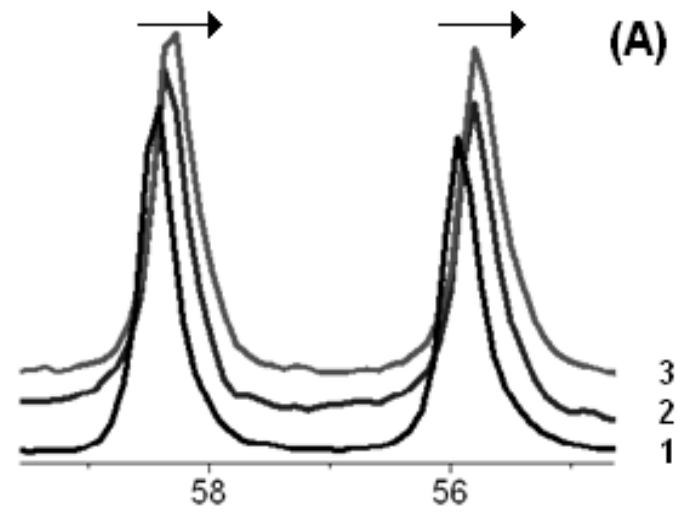

${ }^{13} \mathrm{C}$ Chemical Shift (ppm)

(B)

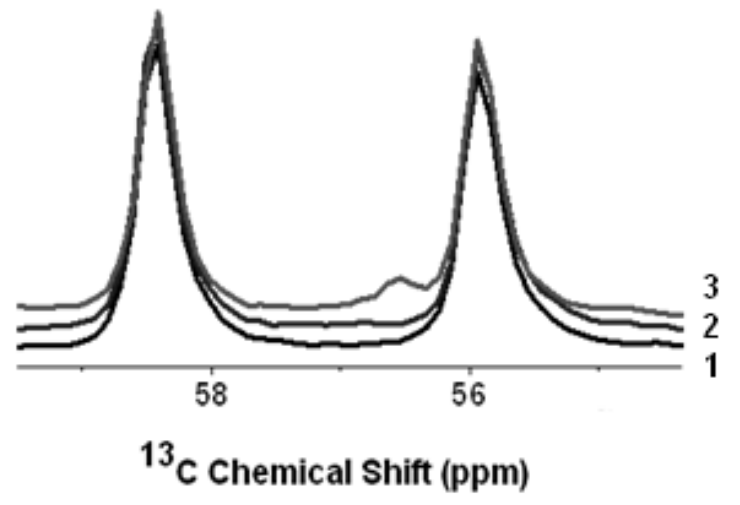

Figure 7. ${ }^{13} \mathrm{C}$ NMR spectra of acrylamide based photopolymer containing (A) zeolite Beta and (B) zeolite A nanoparticles: 1 - undoped acrylamide based photopolymer, 2 - zeolite doped photopolymer (outside grating), 3 - zeolite doped photopolymer (inside grating). Spatial frequency $1000 \mathrm{lmm}^{-1}$ and recording energy of $600 \mathrm{mJcm}^{-2}$ 

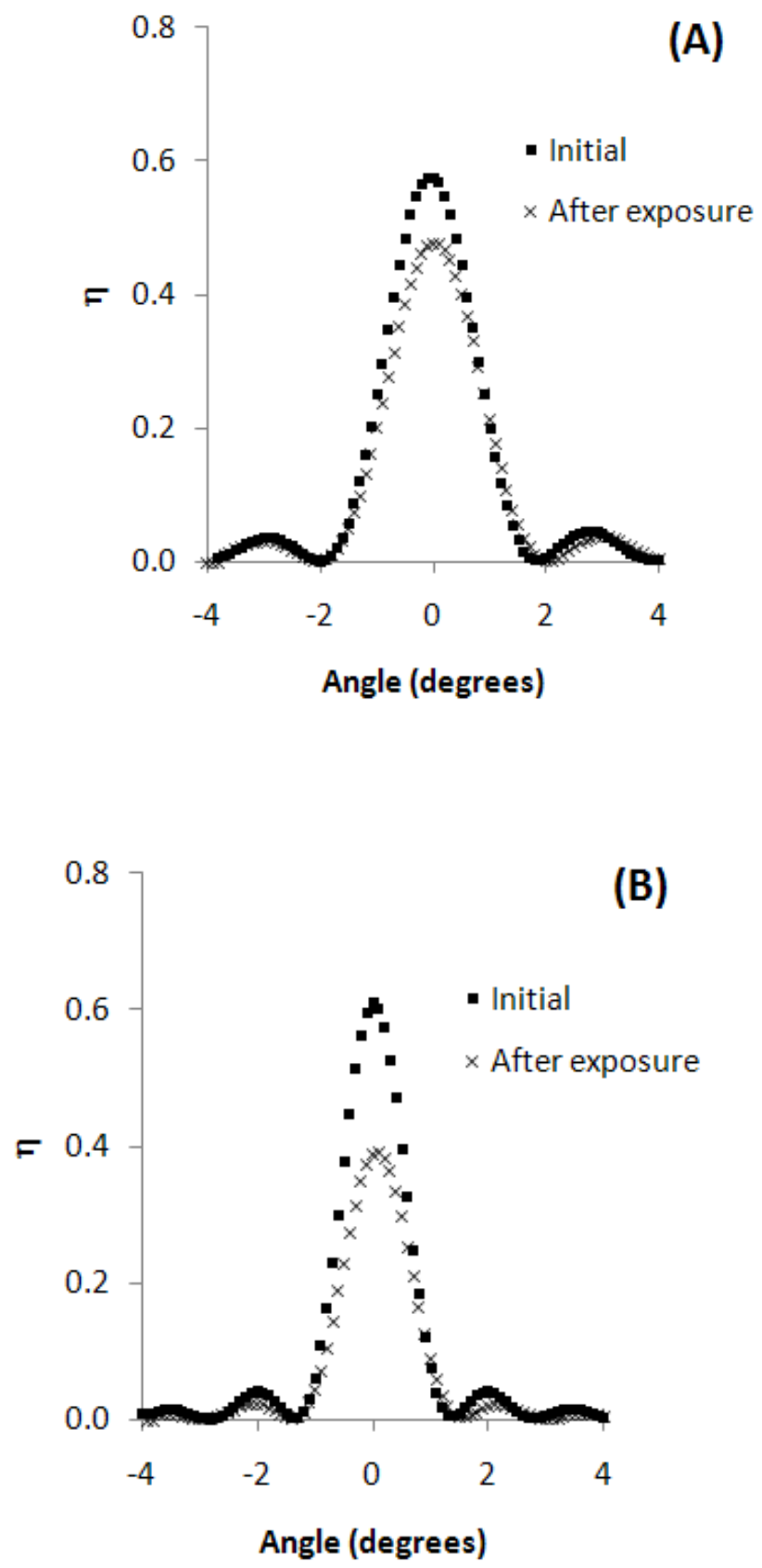

Figure 8. Angular selectivity curves measured before and after exposure to toluene (19ppm) for the highest change in the diffraction efficiency observed: (A) Undoped photopolymer; (B) Photopolymer containing Beta nanoparticles 


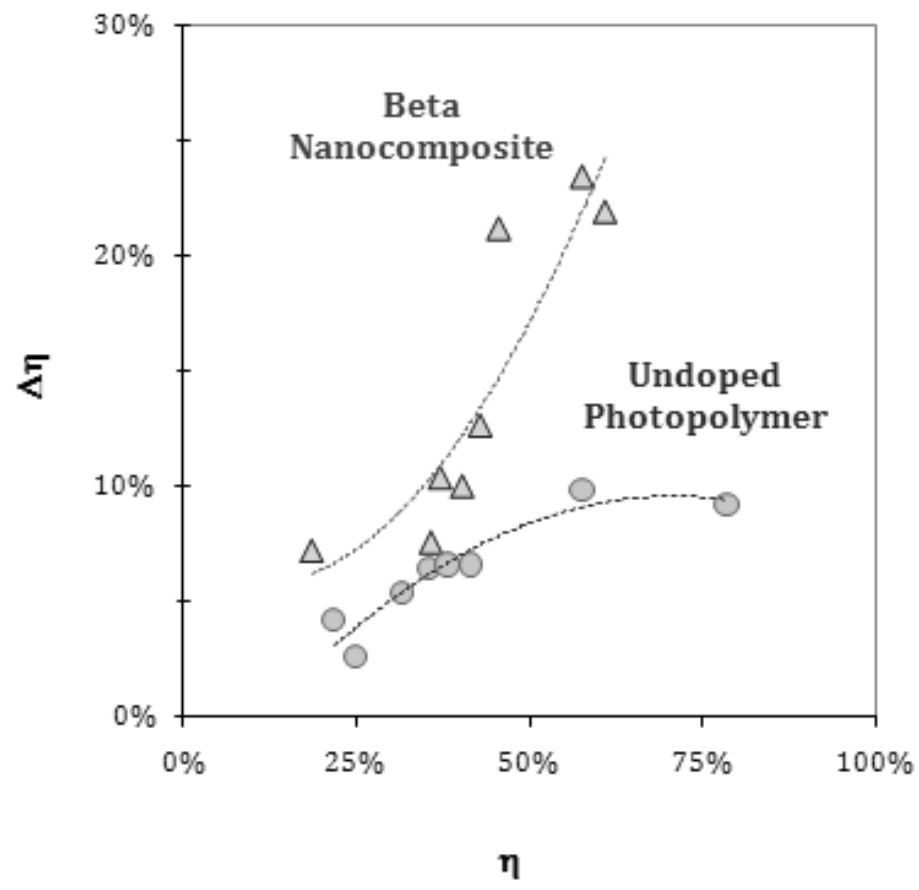

Figure 9. Change in diffraction efficiency of a grating exposed to toluene for Beta photopolymerizable nanocomposite (diamonds) and undoped acrylamide based photopolymer (circles) 